\title{
Análise espacial da mortalidade por acidentes de motocicleta nos municípios do Brasil
}

\author{
Spatial analysis of motorcycle-related mortalities in Brazilian \\ municipalities
}

Pedro Cisalpino Pinheiro (https://orcid.org/0000-0002-6954-1708) ${ }^{1}$

Bernardo Lanza Queiroz (https://orcid.org/0000-0002-2890-1025) ${ }^{1}$

${ }^{1}$ Centro de

Desenvolvimento e Planejamento Regional de Minas Gerais, Universidade Federal de Minas Gerais. Av. Antônio Carlos 6627, Pampulha. 31270-901 Belo Horizonte MG Brasil. ppinheiro@ cedeplar. ufmg.br

\begin{abstract}
Analysis of the distribution of motorcycle-related mortality rates in Brazilian municipalities is fundamental to understand and seek to minimize the occurrence of this growing phenomenon. The main objective of this work is to analyze the spatial distribution of motorcycle rider mortality rates in Brazil, based on more robust and reliable estimates. An attempt was also made to identify the presence of spatial clusters in the distribution of such mortality rates in given municipalities. The rates were estimated based on the average number of motorcyclist deaths recorded in the years 2014, 2015 and 2016. These rates were then directly standardized and graduated based on the local empirical Bayesian estimator. A Local Indicator of Spatial Autocorrelation (LISA) indicated the presence of spatial patterns. The Northeast and Mid-West regions concentrated most of the municipalities with high mortality rates as well and most of the clusters of municipalities with a high-high distribution pattern. Graduated Bayesian estimation was effective to deal with the occurrence of extreme values, thereby improving the reliability of the estimates and enhancing the visualization of the rates on the map.

Keywords Mortality, Motorcycles, Municipalities, Spatial analysis
\end{abstract}

Resumo $A$ análise da distribuição da mortalidade de motociclistas nos municípios brasileiros é fundamental para o entender e tentar minimizar a ocorrência deste crescente fenômeno. O principal objetivo do presente trabalho é analisar a distribuição espacial das taxas de mortalidade de ocupantes de motocicletas nos municipios brasileiros, a partir de estimativas mais robustas e confiáveis. Adicionalmente, pretendeu-se identificar a presença de padrões espaciais na distribuição das mesmas. As taxas brutas foram construídas com base no número de óbitos de motociclistas considerando a média das ocorrências nos anos de 2014, 2015 e 2016. Estas taxas foram padronizadas diretamente e suavizadas utilizando o estimador bayesiano empírico local. A identificação da presença de padrões espaciais foi realizada a partir de um indicador local de autocorrelação espacial (LISA). As taxas de mortalidade mais elevadas foram observadas em municípios da região Nordeste e Centro-Oeste. Estas regiões concentraram, também, a maior parte aglomerados de municípios com padrão alto-alto na distribuição das taxas. A suavização bayesiana se mostrou eficiente para lidar com os valores extremos, ampliando a confiabilidade das estimativas e melhorando a visualização das mesmas no mapa.

Palavras-chave Mortalidade, Motocicletas, Municípios, Análise espacial 


\section{Introdução}

Nas últimas décadas, no Brasil, houve aumento expressivo do número de acidentes relacionados às ocorrências de trânsito e à utilização de motocicletas $^{1-6}$. Dada à natureza do veículo, muitos destes eventos resultam em morte, em ferimentos graves, ou em sequelas permanentes ${ }^{7-10}$. Os maiores riscos de mortalidade por acidente de motocicleta são de jovens entre 15 e 49 anos implicando em um grande custo social. De acordo com estimativas do projeto Carga Global de Doenças, os acidentes de motocicleta correspondem a 1,69\% do total de DALY, 2,58\% para os homens, no Brasil. No mesmo período, houve crescimento significativo da frota de motocicletas no Brasil, passando de 2,5 milhões (1998) para 21,8 milhões, em $2017^{11}$. No país, a taxa de mortalidade no Brasil por acidentes de motocicleta passou de 0,5 (por 100. 000), em 1996, para 4,5, em $2009^{3}$, e 5,9, em 2015, com base nos dados do Datasus.

A mortalidade relacionada às motocicletas aumentou em todos os estados ${ }^{2,3}$. O Retrato da Segurança Viária - $2015^{12}$ mostra que no Brasil, em 2013, as mortes de motociclistas representavam a maior participação no total de mortes de acidentes de transporte (AT), 37\%. Na região Nordeste, observa-se a proporção mais significativa dos óbitos de motociclistas em relação ao total dos AT (49\%). O Brasil, em relação aos demais países das Américas, ao lado de Colômbia e Paraguaii ${ }^{13}$, apresenta taxas de mortalidade de ocupantes de motocicletas elevadas. Os acidentes de transporte no Brasil representam 4,51\% dos Disability Adjusted Lost Years (DALY). Dentre as causas externas, estes acidentes representam cerca de $27 \%$ dos DALY, sendo um dos principais responsáveis pela sobremortalidade masculina ${ }^{14}$.

$\mathrm{O}$ aumento das ocorrências de óbitos, bem como da distribuição dos mesmos no território, significa a ampliação dos custos relacionados aos mesmos $^{15-18}$. A OMS afirma que, entre impactos econômicos diretos e indiretos, o custo associado aos AT, em nível nacional, está entre 1 e $2 \%$ do $\mathrm{PIB}^{16}$. No Brasil, há três estimativas realizadas pelo IPEA $^{19-21}$. Nas aglomerações urbanas, em 2001, os custos dos acidentes foram estimados em $\mathrm{R} \$ 5,3$ bilhões $(0,4 \% \text { do PIB })^{19}$ e, nas rodovias, entre julho de 2004 e junho 2005, em, aproximadamente, $\mathrm{R} \$ 22$ bilhões $^{20}$, estimativas recentes do IPEA indicam impacto econômico de 40 bilhões de reais com acidentes de trânsito ${ }^{21}$.

O período de crescimento da frota de motocicletas, no Brasil, coincide, em grande medida, com um importante momento de crescimento econômico ${ }^{22}$. Ciclos de aumento da atividade produtiva são, de modo geral, associados ao aumento da mortalidade por acidentes de transporte ${ }^{23-26}$. Na Europa, por exemplo, um dos efeitos da crise econômica de 2008 foi a redução da mortalidade por $\mathrm{AT}^{27}$.

A mortalidade por acidentes de transporte tem relação, também, com o processo de desenvolvimento econômico ${ }^{28-31}$. Essa associação, no entanto, não é linear. Para níveis relativamente baixos de desenvolvimento, o aumento da renda apresenta associação positiva com a mortalidade por AT. A partir de um determinado ponto, o aumento da renda passa a representar um fator protetivo ${ }^{28}$. Os efeitos protetivos do aumento da prosperidade se manifestam através de mecanismos de adaptação relacionados à prevenção, melhoria da assistência médico-hospitalar e melhoria da infraestrutura ${ }^{28}$. Law et al. ${ }^{29}$ identificaram a mesma relação para a mortalidade por acidentes de motocicleta.

O presente trabalho tem como objetivo principal produzir estimativas e analisar a distribuição espacial das taxas de mortalidade por acidentes de motocicleta nos municípios brasileiros, tendo como base o ano de 2015. Estudos sobre acidentes de transito no Brasil se concentram em análises para todo o país, grandes regiões e localidades específicas $^{1-6,32,33}$. Não há muitos trabalhos buscando estimar a mortalidade para pequenas áreas e realizar uma análise espacial para essa causa específica. Uma das hipóteses de trabalho é que a taxa de mortalidade por acidente de motocicleta, no Brasil, reflita, em alguma medida, as diferenças no estágio de desenvolvimento dos municípios, bem como que tenha relação com os ciclos de aquecimento da economia. O Brasil é um país marcado por grande desigualdade ${ }^{34}$, trabalha-se com a hipótese de que os diferenciais na mortalidade de motociclistas sejam, também, bastante expressivos. A análise espacial será útil na identificação de aglomerações de municípios com padrões específicos de distribuição das taxas. O objetivo é investigar se a localização geográfica, ou seja, questões de natureza espacial, e o padrão recente de desenvolvimento têm influência sobre a mortalidade relacionada aos acidentes de motocicleta.

\section{Métodos}

\section{Dados}

O número de mortes pela causa "Motociclista traumatizado em um acidente de transporte" (V20-V29) (Classificação Internacional de Do- 
enças -CID-10), por grupo de idade e sexo, de acordo com o município de residência, foi coletado do Sistema de Informações de Mortalidade (SIM) disponível no Datasus, para os anos de 2014, 2015 e 2016. Os óbitos que apresentavam idade, sexo ou município ignorado foram realocados de acordo com distribuição proporcional de cada uma dessas variáveis. Considerando os três anos, foram registrados 24 óbitos com idade ignorada $(0,07 \%), 44$ com município ignorado - em 9 a idade também era ignorada $(0,14 \%)$ e cinco registros de sexo desconhecido $(0,02 \%)$. Uma vez redistribuídos os óbitos, foi calculado o número médio de mortes registradas, em cada município, considerando os três anos em questão. Esse é o numerador utilizado na construção das taxas. No denominador, foi considerada a população de cada município, por sexo e idade, segundo estimativa da população para 2015 realizada pelo Ministério da Saúde ${ }^{35}$.

As taxas brutas de mortalidade, por sexo, foram padronizadas pelo método direto ${ }^{36,37}$, tendo como base a distribuição etária da população brasileira estimada para o ano de 2015. No artigo, focamos a análise na população masculina que, em cada um dos três anos em questão, respondeu por $89 \%$ do total das mortes desse estudo.

\section{Suavização bayesiana}

A identificação de padrões espaciais de distribuição de uma doença é fundamental para o desenvolvimento de medidas de prevenção e controle $^{38}$. No entanto, as taxas brutas observadas em municípios pequenos são pouco indicadas para a realização desse tipo de análise, uma vez que podem sofrer influência de flutuação aleatória e complicações de cálculos em função dos pequenos números ${ }^{38-43}$.

Por serem pouco populosos, afirmam Carvalho et al. ${ }^{38}$, o baixo número de casos observados em um município pequeno pode levar a estimativas pouco representativas, ou mesmo distorcidas, da realizada observada. Para eventos relativamente raros (a morte de um motociclista, por exemplo), muitas vezes, as taxas brutas podem ser iguais a zero, o que não significa dizer que há ausência de risco associado. Em alguns casos, as taxas brutas iguais a zero são reflexos de uma janela temporal que não foi suficientemente longa ${ }^{38}$.

Um segundo problema com as taxas de pequenas áreas é que, como o denominador é pequeno, a variabilidade nas estimativas tende a ser muito grande. Assunção et al. ${ }^{39}$ mostram que, de modo geral, pequenas regiões apresentam uma menor população sob risco, e, assim, o acréscimo ou decréscimo de uma ocorrência no numerador pode representar grande variação nas estimativas. O grau de variabilidade aleatória está associado ao tamanho das unidades geográficas analisadas ${ }^{39}$. Para lidar com este tipo de problema, estes autores destacam que métodos bayesianos empíricos ou bayesianos são possíveis soluções e alternativas.

Um meio de incorporar a localização no estimador bayesiano empírico é através da definição de estruturas de vizinhança para cada área. A definição dos vizinhos de cada observação servirá de base para a definição dos parâmetros à priori. Ou seja, as taxas das pequenas áreas, principalmente, irão convergir em direção à média das taxas de seus vizinhos ${ }^{40}$. Em relação às abordagens globais, a estimativa que considera a média local dos vizinhos apresentará uma suavidade espacial mais próxima da realidade dos eventos de interesse $^{38}$.

Neste trabalho, optamos pela utilização dos estimadores bayesianos empíricos de James-Stein, operacionalizados por Marshall ${ }^{40}$. Nesse modelo, os estimadores são definidos de acordo com as equações 1 e 2 .

$$
\begin{aligned}
& \hat{\theta}=\widetilde{m}+\widetilde{C}_{i}\left(x_{i}-\tilde{m}\right) \\
& \widetilde{C}=s^{2}-\frac{\widetilde{m}}{\bar{n}}\left(s^{2}-\tilde{m} / \bar{n}+\frac{\bar{n}}{n}\right)
\end{aligned}
$$

Onde, $\hat{\theta}=\tilde{m}$, quando $s^{2}<\tilde{m} / \bar{n}$. $\widetilde{C}$ é o fator de contração; $\tilde{m}$ é a média de $\mathrm{x}_{\mathrm{i}}$; $\mathrm{x}_{\mathrm{i}}$ é a taxa bruta em i; $\mathrm{s}^{2}$ é a variância amostral de $\mathrm{x}_{\mathrm{i}}$; e $\mathrm{n}_{\mathrm{i}}$ é o número de pessoas-ano sob risco ${ }^{40}$.

Muitas vezes é razoável considerar que áreas mais próximas apresentam padrões de doença similares. Assim, a chamada abordagem local utiliza as informações dos vizinhos de cada observação como os parâmetros prévios para o ajuste de $\theta$. Neste sentido, $\theta$ é estimado pela contração de $x_{i}$ em direção à média observada em seus vizinhos $^{40}$.

Para a definição da vizinhança, optamos pela utilização de um número definido de k-vizinhos $(\mathrm{k}=8)$ mais próximos. Para a avaliação da efetividade da estrutura de vizinhança, analisamos a redução da variabilidade das taxas suavizadas em relação às taxas padronizadas por meio de um gráfico de dispersão. Ao considerar as informações dos vizinhos, a suavização bayesiana incorpora um componente espacial para a construção das estimativas. 


\section{Autocorrelação espacial}

A autocorrelação espacial é uma medida de grau de influência de uma determinada variável, em uma localização específica, sobre a mesma variável em localidades próximas. Ou seja, a ausência de autocorrelação espacial indica que a localização não tem influência no valor de uma variável observada em um determinado ponto. Quando há a presença de autocorrelação espacial, o interesse volta-se para os casos em que valores elevados foram observados próximos a outros locais com o indicador de interesse também altos, ou baixos valores próximos a locais com o indicador também baixo. Da mesma forma, locais com nível alto da variável de interesse próximo a pontos com valores baixos (ou vice-versa) ${ }^{44}$. Nestas situações, a localização tem influência sobre o valor observado na variável de interesse.

Para identificação do espaço como variável relevante nas taxas de mortalidade utilizamos uma variação do I de Moran que adota uma abordagem bayesiana empírica, proposta por Assunção e Reis ${ }^{45}$. O Empirical Bayes Index (EBI) além de melhorar a confiabilidade do teste de hipótese, é mais robusto em função do efeito de contração das taxas brutas. No lugar da utilização das taxas de cada área, $\mathrm{p}_{\mathrm{i}}$, os autores propõem a utilização do desvio da média marginal estimada, padronizada por uma estimativa do desvio padrão:

$$
z_{i}=p_{i}-b / \sqrt{v_{i}}
$$

Onde, $\mathrm{p}_{\mathrm{i}}$ é a taxa na área $\mathrm{i}$, b e $\mathrm{v}_{\mathrm{i}}$ representam, respectivamente, a esperança e a variância marginal de $\mathrm{p}_{\mathrm{i}}$, tal qual apresentadas por $\mathrm{Marshall}^{40}$. O EBI é, então, definido por:

$$
\mathrm{EBI}=\frac{m}{\sum w_{i j}} \frac{\sum w_{i j} z_{i} z_{j}}{\sum\left(z_{i} \bar{z}\right)^{2}}
$$

Onde, $\mathrm{w}_{\mathrm{ij}}$ é o peso espacial atribuído às áreas i e j; $z_{i}$ é o desvio à média observado em $i, z_{j}$ é o desvio à média em j; e $\bar{z}$ é a média dos desvios médios; e m é o número de áreas.

A análise espacial dos municípios foi feita a partir do EBI, um indicador local (LISA) que permite identificar padrões de associação espacial. A análise do LISA permitiu classificar os municípios brasileiros com base na significância dos índices locais em quatro clusteres. Os cluster são: alta/alta e baixa/baixa que significa que possuem associação positiva, isto é a localização possui vizinhos com valores próximos de mortalidade pela causa estudada, e alta/baixa e baixa/ alta, indicam associação negativa, ou seja, que a localização possui vizinhos distintos de mortalidade, ou seja, localidades com mortalidade baixa, mas vizinha de localidades de mortalidade alta ou vice-versa.

\section{Resultados}

A Tabela 1 apresenta a média das taxas de mortalidade padronizadas $\left(\mathrm{TBM}^{\mathrm{s}}\right)$ dos municípios (por 100. 000 habitantes), por região, para os homens onde são destacados, também, o desvio padrão e os valores máximos e mínimos observados em cada região. As regiões Nordeste $(24,3)$, Centro-Oeste $(20,4)$ e Norte $(19,4)$, apresentaram as maiores médias das taxas de mortalidade de motociclistas do sexo masculino. As taxas padronizadas nestas três regiões foram mais que duas vezes superiores que a região com a taxa mais baixa (Sudeste).

Como as taxas padronizadas são afetadas pelas flutuações aleatórias e pelo pequeno número de casos, uma estimativa mais robusta foi obtida usando o modelo bayesiano empírico. A Figura 1 apresenta as taxas de mortalidade suavizadas pelo método e permite visualizar, com nitidez, a extensão da mancha de municípios de mortalidade mais elevada, desde o estado do Mato Grosso do Sul, até o estado de Sergipe, passando por diversos estados das regiões Centro-Oeste, Norte e Nordeste. Notadamente, as regiões com as maiores $\mathrm{TBM}^{\mathrm{s}}$ (Tabela 1). Chama a atenção que os estados do Maranhão e Piauí parecem concentrar os municípios com as taxas de mortalidade de motociclistas mais elevada.

As regiões Sul e Sudeste, que apresentam maior desenvolvimento econômico do país ${ }^{46}$, destacam-se pelo grande número de municípios com taxas de mortalidade mais baixas. Chama a atenção, no entanto, na região Sudeste, a alta concentração de municípios com mortalidade de motociclistas elevada na região norte do Rio de Janeiro e no sul do estado do Espírito Santo, bem como parte de Minas Gerais, próximos às fronteiras com esses dois estados. Os municípios que formam os Vales do Jequitinhonha e do Mucuri, em Minas Gerais, estão entre aqueles com TBM ${ }^{\mathrm{s}}$ mais elevadas. Muitos destes apresentam baixos níveis de desenvolvimento socioeconômico. $\mathrm{Na}$ região Sul, as regiões com as taxas bayesianas mais elevadas foram observadas no oeste e norte do Paraná. Em Santa Catariana, praticamente no estado como um todo, as taxas apresentadas na Figura 1 estão entre as mais elevadas da região.

Um segundo ponto de destaque na Figura 1 é a expressiva diferença do nível das taxas obser- 
Tabela 1. Taxa Bruta Padronizada Média, desvio padrão, máxima e mínima, das taxas padronizadas de mortalidade (por 100.000 habitantes) de motociclistas por município, Brasil e grandes regiões.

\begin{tabular}{lrrrrr}
\hline & Observações & Taxa Padronizada & Desvio Padrão & Taxa Máxima & Taxa Mínima \\
\hline Brasil & 5570 & 16,48 & 18,76 & 206,13 & 0,00 \\
Norte & 450 & 19,42 & 19,75 & 128,19 & 0,00 \\
Nordeste & 1794 & 24,26 & 22,74 & 206,13 & 0,00 \\
Sudeste & 1668 & 9,38 & 11,64 & 93,49 & 0,00 \\
Sul & 1191 & 12,03 & 15,07 & 111,42 & 0,00 \\
Centro-Oeste & 467 & 20,42 & 16,97 & 125,81 & 0,00 \\
\hline
\end{tabular}

Fonte: Datasus, 2014, 2015 e 2016.

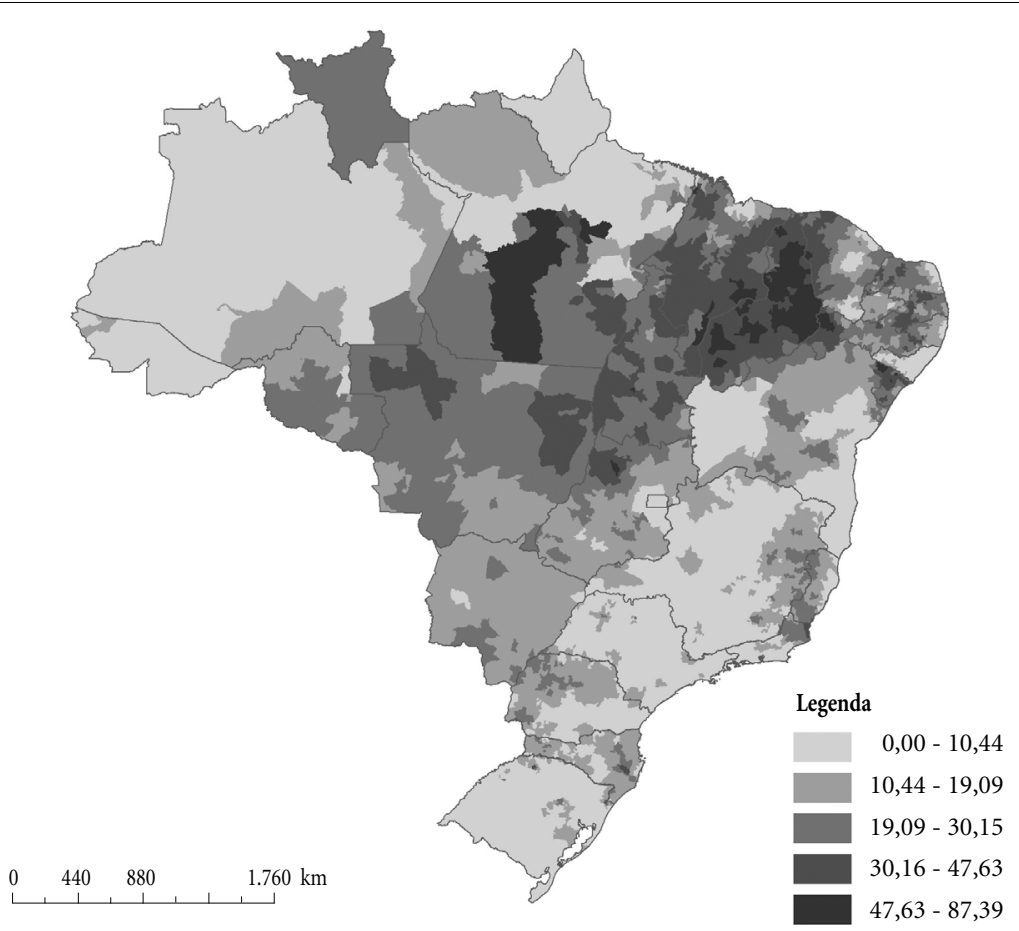

Figura 1. Taxas de mortalidade suavizadas (EBS), por município, homens, Brasil, 2015, por 100.000 habitantes.

Fonte: Datasus, 2014,2015 e 2016.

vadas a partir da suavização bayesiana, quando comparada às taxas padronizadas. Considerando a população masculina (Tabela 1), a $\mathrm{TBM}^{\mathrm{s}}$ mais elevada era de 206,1 (por 100. 000), por sua vez, a taxa de mortalidade suavizada mais elevada registrada foi equivalente a 87,4 .

A Figura 2 apresenta os padrões de autorcorrelação espacial usando o LISA. Na região Norte, é possível identificar um grande aglomerado de municípios de padrão baixo-baixo - municípios com baixas taxas de mortalidade, cujos vizinhos apresentam também taxas baixas nos estados do Acre, Amazonas, região marcada pela presença da Floresta Amazônica. Observa-se, também, um aglomerado de padrão alto-alto no sul do Pará, essa região já se destacava entre aquelas com elevadas taxas de mortalidade.

Na região Nordeste, localiza-se o aglomerado de padrão alto-alto que agrega o maior número de municípios com elevada mortalidade, cercado por vizinhos com a mesma característica. O referido cluster está concentrado, principalmente, nos estados do Maranhão, Piauí e Tocantins. Na Bahia, no sentido oposto aos demais estados da região, há a presença de aglomerados de municípios com baixas taxas de mortalidade de ocu- 


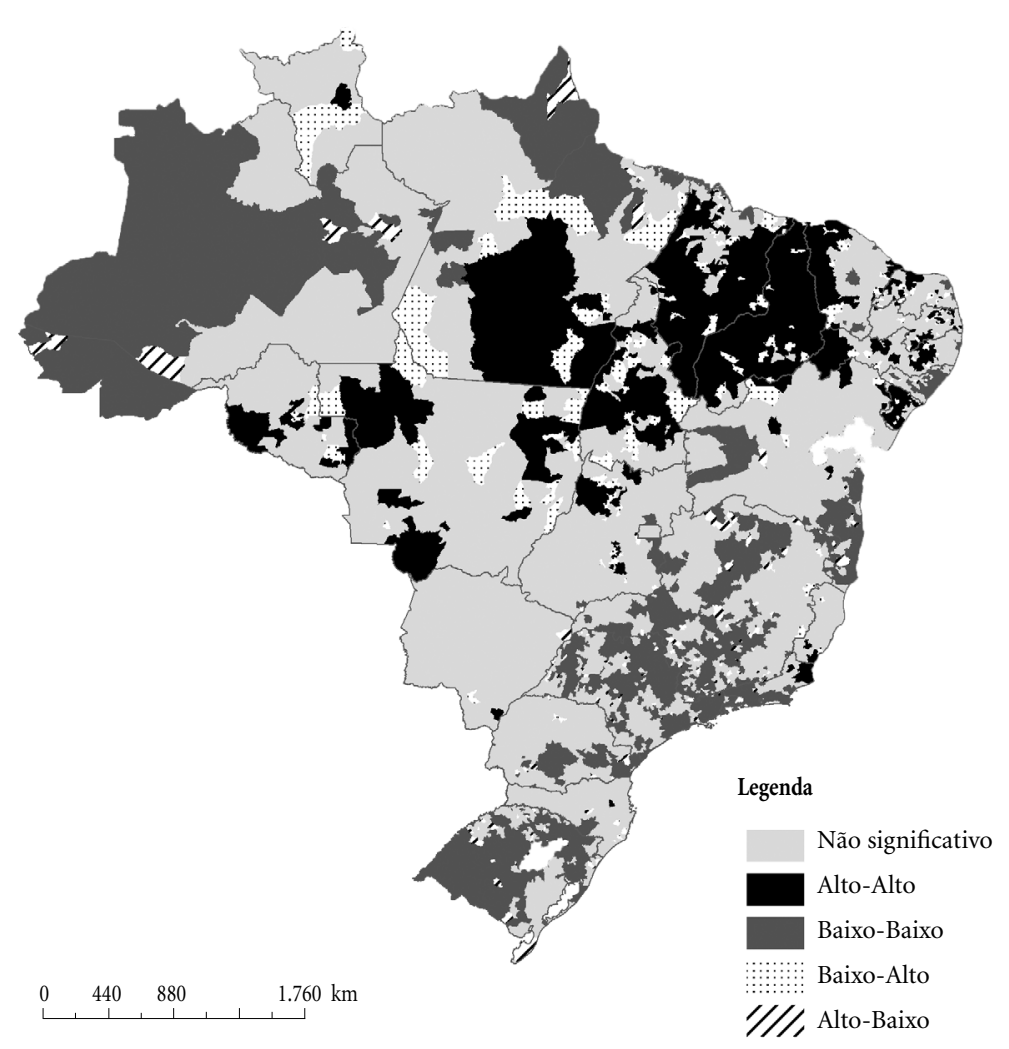

Figura 2. LISA cluster map (EBI) das taxas de mortalidade padronizadas, homens, Brasil, 2015, por 100.000 habitantes.

Fonte: Datasus: 2014, 2015, 2016.

pantes de motocicletas (Figura 2). Nas regiões Sul e Sudeste, em praticamente todos os estados, é possível identificar a presença de aglomerados de padrão baixo-baixo. No entanto, observa-se um aglomerado de padrão alto-alto no norte do estado do Rio de Janeiro e no sul do estado do Espírito Santo. Ainda nas regiões Sul e Sudeste merecem destaque a presença de alguns pequenos aglomerados de municípios de alta mortalidade, cujos vizinhos apresentam mortalidade mais baixa. Estes pequenos aglomerados estão espalhados em praticamente todos os estados da dessas regiões.

\section{Discussão}

As metodologias aplicadas nesse artigo possibilitaram obter medidas robustas de mortalidade por acidentes de motocicletas nos municípios brasileiros, em 2015, e analisar o padrão espacial dessa causa de morte no país. Estimativas adequadas de mortalidade e de causas de morte por pequenas áreas são informações fundamentais para planejar medidas de saúde pública na maioria dos países, da mesma forma a avaliação da qualidade dos dados é um passo importante para alcançar altos níveis de qualidade ${ }^{38,42,47}$. Estudos anteriores sobre essa causa de morte se concentraram na análise do país ${ }^{4,5,13}$, grandes regiões ${ }^{4,6,12}$, estados $^{2,3,12}$ e por porte de municípios ${ }^{2}$, ou estudos específicos para alguma localidade ${ }^{1,33,48-52}$. Resultados do projeto Carga Global de Doenças mostram o aumento da mortalidade por acidente de motocicletas em diferentes estados no Brasil, com maiores taxas de mortalidade nas regiões Norte e Nordeste em relação ao Sul e Sudeste do país 9 . Do nosso conhecimento, esse trabalho é um dos primeiros a adotar uma metodologia que possibilite estudar todos os municípios no Brasil 
e analisar de forma mais específica a variabilidade e heterogeneidade do impacto da mortalidade por acidentes de trânsito no país.

A distribuição espacial da mortalidade por acidentes de motocicletas no Brasil destaca uma mancha de municípios que passa, principalmente, pelas regiões Norte, Nordeste e Centro-Oeste. Segundo Martins et al. ${ }^{3}$, a explicação pode ter relação com a grande ascensão econômica observada, especialmente nas regiões destacadas, impulsionada pelo surgimento de novas fronteiras agrícolas e do agronegócio. Entre 1990 e 2009, estas regiões ampliaram sua participação relativa no PIB do país ${ }^{46}$. O aumento da renda em tais regiões teria sido acompanhado da compra de um primeiro veículo, muitas vezes, uma motocicleta ${ }^{3}$. Silva et al..$^{53}$ mostram que essas regiões apresentaram taxa de crescimento do número de motocicletas expressiva. A maior parte dos municípios da região Nordeste apresenta uma frota de motocicletas superior à frota de automóveis ${ }^{53}$. A relação entre atividade econômica e mortalidade por acidentes de transporte é pro-cíclica, tendo sido observada em diversos países ${ }^{23-26}$. Considerando os membros da OCDE, por exemplo, a queda de $1 \%$ no desemprego foi associada a um crescimento de $2,1 \%$ na taxa de mortalidade por acidentes de transporte ${ }^{23}$.

Nas últimas décadas, parte das regiões do país que apresentaram maior crescimento da produtividade agrícola estão entre aquelas de mortalidade por acidentes de motocicleta mais elevadas. A chamada região Mapitoba, que compreende os estados do Maranhão, Piaú, Tocantins e Bahia, por exemplo, apresentou, entre 1990 e 2013, crescimento expressivo em sua participação relativa na produção agropecuária. Esta região foi responsável por $8,4 \%$ da produção total de toneladas de soja em 2013. No mesmo período, a região Centro -Oeste (a segunda $\mathrm{TBM}^{\mathrm{S}}$ mais alta) elevou substancialmente sua participação relativa na produção de soja (32,4\% para 46,8\%), de milho $(14,6 \%$ para $44,7 \%)$ e algodão $(10,7 \% \text { para } 65,7 \%)^{54}$.

Muitos dos municípios dos estados do Rio de Janeiro e Espírito Santo estão entre aqueles que apresentaram alta mortalidade de motociclistas (Figura 1). Martins et al. ${ }^{3}$, observam que o crescimento da mortalidade de motociclistas nestes estados pode ser associado ao desenvolvimento da economia petrolífera. No Espírito Santo, por exemplo, Caçador e Monte ${ }^{55}$, com base em dados da Agência Nacional do Petróleo (ANP), salientam que a produção de gás natural e de petróleo cresceu a uma média anual de, respectivamente, $23,9 \%$ e $67 \%$ (2000-2009). A distribuição de royalties de gás e petróleo para os municípios daquele estado passou de R $\$ 10$ milhões, em 2000, para R \$194 milhões, em 2009.

Kopits e Cropper ${ }^{31}$ observam que a mortalidade por AT é consequência da relação veículos por pessoas e da letalidade por veículo. Neste sentido, estes autores enfatizam que a variação da fatalidade depende do número de veículos por habitante, bem como da relação entre o número de veículos e as mortes a eles associadas. Bishiai et al. ${ }^{30}$ destacam que apesar da redução da fatalidade por AT com o aumento da renda, não foi percebida queda no número de acidentes registrados, nem do número de pessoas feridas em função dessas ocorrências. Neste sentido, Noland ${ }^{56}$ observa que o avanço tecnológico médico apresenta impacto significativo na redução da mortalidade relacionada aos meios de transporte.

A região Norte apresentava (em agosto de 2015) a menor proporção de médicos por habitantes (O número de médicos e a quantidade de leitos por região foram coletados no Cadastro Nacional de Estabelecimentos de Saúde (CNES), disponível para consulta no endereço http://datasus. gov. br/) (0,95 médicos por 1.000 habitantes - contra 2,2 da região Sudeste, a mais elevada), assim como menor número de leitos hospitalares por habitante ( 1,81 leitos para cadal. 000 habitantes - a região Sul apresenta a relação de 2,56 ), ao mesmo tempo que estava entre as regiões de mortalidade de motociclistas mais elevadas. No entanto, a relação leitos por habitante, sozinha, não é capaz de explicar os diferenciais entre as taxas de mortalidade entre as regiões. A região Centro-Oeste, por exemplo, é a terceira considerando o número mais elevado de médicos por habitantes $(1,76 / 1.000)$ e a segundo com o maior número de leitos por habitante $(2,6 / 1$. 000), ao mesmo tempo que concentra um número expressivo de municípios com elevada mortalidade de motociclistas.

Outros fatores, além do crescimento econômico e da motorização, podem ser relevantes para explicar a elevada mortalidade de ocupantes de motocicletas. Luz et al. ${ }^{1}$, por exemplo, observaram que na zona rural de Itiúba (BA), $87 \%$ dos condutores de motocicleta declararam não possuir habilitação e $43,7 \%$ não utilizar o capacete. Pordeus et al. ${ }^{57}$ observaram que 55,8\% dos atendimentos em um hospital de Fortaleza haviam sido realizados em motociclistas sem habilitação e 56,9\% não utilizavam capacete. Em Pelotas, $49 \%$ dos motociclistas entrevistados por Seerig et al. ${ }^{52}$ declaram não fechar a alça do capacete de modo adequado. O Inquérito VIVA de 
2014 mostra que nas capitais das regiões Norte e Nordeste uma maior proporção de motociclistas acidentados que não utilizavam capacete e que declararam consumo prévio de bebidas alcoóli$\mathrm{Cas}^{58}$. É bastante provável que estes percentuais sejam ainda mais elevados em municípios menos desenvolvidos do ponto de vista institucional, e, assim, com menor capacidade de fiscalização.

Tay ${ }^{59}$ destaca que, do ponto da análise econômica, o interesse sobre o cumprimento ou não das leis recai sobre os custos percebidos pelos indivíduos de se envolver em alguma atividade ilegal - como conduzir motocicleta sem capacete. Neste sentido, o aumento do custo associado (seja a elevação da severidade da pena ou da probabilidade de apreensão) a um comportamento ilegal resulta em uma redução do mesmo. Para Tay ${ }^{59}$, no campo da segurança de trânsito, o principal papel das leis e da fiscalização é ampliar a certeza de apreensão e de punição.

As evidências apresentadas permitem entender a grande distribuição de municípios com taxas de mortalidade elevada em regiões menos desenvolvidas do país. Além de contar com vias de pior qualidade (que também é um fator determinante para a ocorrência de acidentes) ${ }^{25}$, a capacidade de fiscalização é bastante reduzida. Se mesmo em capitais, como Fortaleza, a participação de motoristas sem habilitação, sem capacete e que conduzem sob o efeito do álcool é bastante relevante, é provável que esses números em municípios menores e com menor capacidade de fiscalização sejam ainda mais significativos.

\section{Colaboradores}

PC Pinheiro trabalhou na concepção, análise e interpretação dos dados, redação do artigo, revisão crítica e aprovação da versão a ser publicada. BL Queiroz trabalhou na análise e interpretação dos dados, redação do artigo, revisão crítica, bem como na aprovação da versão a ser publicada.
A identificação da autocorrelação espacial de padrão alto-alto em boa parte das regiões em que as taxas de mortalidade haviam sido destacadas entre as mais elevadas indica que existem fatores de localização, comuns aos municípios desses aglomerados, que influenciam os níveis das taxas nessas regiões. Convém ressaltar que, como aponta $\mathrm{Anselin}^{44}$, a identificação da presença de autocorrelação espacial não é capaz de informar o porquê da existência dos clusters, nem mesmo os fatores que determinam sua força e forma. Em grande medida, os aglomerados de padrão alto-alto coincidem com os aglomerados de risco de mortalidade elevado identificados por Morais Neto et al. ${ }^{2}$.

A mortalidade crescente por causas relacionadas à utilização de motocicleta deve ser entendida como um problema relevante no Brasil. Da mesma forma, o impacto econômico e de saúde em decorrência das internações e assistência aos acidentados. Neste sentido, demanda informações de qualidade para compreender o cenário em que está posto e, consequentemente, para planejar possíveis soluções. A utilização da suavização bayesiana mostrou-se uma estratégia eficiente para lidar com os problemas de flutuação aleatória, controlando, em boa medida, os valores extremos observados em alguns municípios. Da mesma forma, os mapas com as taxas suavizadas representaram ganhos expressivos em termos de visualização, mostrando-se como uma ferramenta importante para análise da distribuição das taxas no espaço.

\section{Agradecimentos}

Esse trabalho é parte do projeto "Estimativas de Mortalidade e Construção de Tabelas de Vida para Pequenas Áreas No Brasil", financiado pelo CNPq. 


\section{Referências}

1. Luz CM, Brito E, Gortado G, Júnior J, Tonhá MC, Neto P. Perfil do condutor de motocicletas em zona rural do interior da Bahia. Revista da Abramet 2009; 27(1):22-31.

2. Morais Neto OL, Montenegro MMS, Monteiro RA, Siqueira Júnior JB, Silva MMA, Miranda LOM, Malta DC, Silva Júnior JB. Mortalidade por Acidentes de Transporte Terrestre no Brasil na última década: tendência e aglomerados de risco. Cien Saude Colet 2012; 17(9):2223-2236.

3. Martins ET, Boing AF, Peres MA. Mortalidade por acidentes de motocicleta no Brasil: análise de tendência temporal, 1996-2009. Rev Saude Publica 2013; 47(5):931-941.

4. Chandran A, Sousa TRV, GuoY,Bishai D, Pechansky F, The Vida No Trânsito Evaluation Team. Road Traffic Deaths in Brazil: Rising Trends in Pedestrian and Motorcycle Occupant Deaths. Traffic Inj Prev 2012; 13(Supl. 1):11-16

5. Bachierri G, Barros AJD. Acidentes de trânsito no Brasil de 1998 a 2010: muitas mudanças e poucos resultados. Rev Saude Publica 2011; 45(5):949-963.

6. Morais Neto OL, Andrade AL, Guimarães RA, Mandacarú PMP, Tobias GC. Regional disparities in road traffic injuries and their determinants in Brazil, 2013. Int J Equity Health 2016; 15(1):142

7. Miki N, Martimbianco ALC, Hira LT, Lahoz GL, Fernandes HJA, Reis FB . Profile of trauma victims of motorcycle accidents treated at hospital São Paulo. Acta Ortop Bras 2014; 22(4):219-222.

8. Blincoe L J, Miller T R, Zaloshnja E, Lawrence BA. The economic and societal impact of motor vehicle crashes, 2010. Washington: National Highway Traffic Safety Administration; 2015. (Report No. DOT HS 812 013).

9. Miller TR. Costs and consequences of U. S. roadway crashes. Accid Anal Prev 1993; 25(5):593-607.

10. Ladeira RM, Malta DC, Morais Neto OL, Montenegro MMS, Soares Filho AM, Vasconcelos CH, Mooney M, Naghavi M. Acidentes de transporte terrestre: estudo Carga Global de Doenças, Brasil e unidades federadas, 1990 e 2015. Revista Brasileira de Epidemiologia 2017; 20(Supl. 1):157-170.

11. Departamento Nacional de Trânsito (Denatran). Frota de veículos. Brasília: Denatran; 2017. [acessado 2017 Out 21]. Disponível em: http://www. denatran. gov. br/frota. htm

12. AMBEV S.A. Retrato da Segurança Viária no Brasil 2015. Brasília: Grupo Máquina Pr; 2016.

13. Rodrigues EMS, Villaveces A, Sanhueza A, EscamillaCejudo JA. Trends in fatal motorcycle injuries in the Americas, 1998-2010. Int J Inj Contr Saf Promot 2014; 21(2):170-180.

14. Leite IC, Valente JG, Schramm JMA, Daumas RP, Rodrigues RN, Santos MF, Oliveira AF, Silva RS, Campos MR. Carga de doença no Brasil e suas regiões, 2008. Cad Saude Publica 2015; 31(7):1551-1564.

15. Gururaj G. Road traffic deaths, injuries and disabilities in India: Current scenario. Natl Med J India 2008; 21(1):14-20.

16. World Health Organization (WHO). Global status report on road safety 2013. Geneva: WHO; 2013

17. World Health Organization (WHO). Global status report on road safety 2015. Geneva: WHO; 2015
18. Mohan, D. Social Cost of Road Traffic Crashes in India. Proceedings First Safe Community Conference on Cost of Injury, Viborg, Denmark, October 2002. p. 33-38.

19. Instituto de Pesquisas Econômicas Aplicadas (IPEA). Impactos sociais e econômicos dos acidentes de trânsito nas aglomerações urbanas brasileiras. Brasília: IPEA, ANTP; 2003.

20. Instituto de Pesquisas Econômicas Aplicadas (IPEA). Impactos sociais e econômicos dos acidentes de trânsito nas rodovias brasileiras. Brasília: IPEA, DENATRAN, ANTP; 2006.

21. Instituto de Pesquisa Econômica Aplicada (IPEA), Polícia Rodoviária Federal (PRF). Acidentes de Trânsito nas Rodovias Federais Brasileiras: Caracterização, Tendências e Custos para a Sociedade. Relatório de Pesquisa. Brasília: IPEA, PRF; 2015.

22. Baltar P. Crescimento da economia e mercado de trabatho no Brasil. Brasília: IPEA; 2015.

23. Gerdtham U, Ruhm C. Deaths Rise in Good Economic Times: Evidence From the OECD. Econ Hum Biol 2006; 4(3):298-316.

24. Ruhm CJ. Economic conditions and alcohol problems. J Health Econ 1995; 14(5):583-603

25. Hakim S, Shefer D, Hakkert AS, Hocherman I. A critical review of macro models for road accidents. Accid Anal Prev 1991; 23(5):379-400.

26. Scuffham PA, Langley JD. A model of traffic crashes in New Zealand. Accid Anal Prev 2002; 34(5):673-687.

27. Stuckler D, Basu S, Suhrcke M, Coutts A, McKee M. Effects of the 2008 recession on health: a first look at European data. Lancet 2011; 378(9786):124-125.

28. van Beeck EF, Borsboom GJ, Mackenbach JP. Economic development and traffic accident mortality in the industrialized world, 1962-1990. Int J Epidemiol 2000; 29(3):503-509.

29. Law TH, Noland RB, Evans AW. Factors associated with the relationship between motorcycle deaths and economic growth. Accid Anal Prev 2008; 41(2):234240.

30. Bishai D, Quresha A, James P, Ghaffarc A. National road casualties and economic development. Health Econ 2006; 15(1):65-81.

31. Kopits E, Cropper M. Traffic fatalities and economic growth. Accid Anal Prev 2005; 37(1):169-178.

32. Andrade SSCA, Mello-Jorge MHP. Mortality and potential years of life lost by road traffic injuries in Brazil, 2013. Rev Saude Publica 2016; 50:59

33. Montenegro MMS, Duarte EC, Prado RR, Nascimento AF. Mortalidade de motociclistas em acidentes de transporte no Distrito Federal, 1996 a 2007. Rev Saude Publica 2011; 45(3):529-538.

34. United Nations Development Programme (UNDP). Human Development Report 2016. Ney York: UNDP; 2016.

35. Brasil. Ministério da Saúde (MS). Estimativa da população por sexo e idade, 2015. Brasília: MS; 2018.

36. Preston SH, Hauveline P, Guillot M. Demography. Measuring and Modeling population processes. Malden: Blackwell Pub.; 2001.

37. Wachter KW. Essential demographic methods. Harvard: Harvard University Press; 2014. 
38. Carvalho AXY, Silva GDM, Almeida Júnior GR, Albuquerque PHM. Taxas bayesianas para o mapeamento de homicídios nos municípios brasileiros. Cad Saude Publica 2012; 28(7):1249-1262.

39. Assunção RM, Barreto SM, Guerra HL, Sakurai M. Mapas de taxas epidemiológicas: uma abordagem Bayesiana. Cad Saude Publica 1998; 14(4):713-723.

40. Marshall RJ. Mapping disease and mortality rates using empirical Bayes estimators. J R Stat Soc Ser C Appl Stat 1991; 40(2):283-294.

41. Souza WV, Barcellos CC, Brito AM, Carvalho MS, Cruz OG, Albuquerque, MFM, Alves KR, Lapa TM. Aplicação de modelo Bayesiano empírico na análise espacial da ocorrência de hanseníase. Rev Saude Publica 2001; 35(5):474-480

42. Cavalini LT, Leon ACMP. Correção de sub-registros de óbitos e proporção de internações por causas mal definidas. Rev Saude Publica 2007; 41(1):85-93.

43. Justino JR, Freire FHMA, Lucio PS. Estimação de sub -registros de óbitos em pequenas áreas com os métodos bayesiano empírico e algoritmo EM. Rev bras estud popul 2012; 29(1):87-100.

44. Anselin L. Spatial data analysis with gis: an introduction to application in the social sciences. Buffalo: $\mathrm{Na}-$ tional Center for Geographic Information and Analysis; 1992. [Technical Report 92-10].

45. Assunção R, Reis EA. A new proposal to adjust Moran's I for population density. Stat Med 1999; 18(16):2147-2161

46. Monteiro Neto A. Desigualdades regionais no Brasil: Características e tendências recentes. Boletim regional, urbano e ambiental 2014; 9:67-81.

47. Carvalho MS, Souza-Santos R. Análise de dados espaciais em saúde pública: métodos, problemas, perspectivas. Cad Saude Publica 2005; 21(2):361-378.

48. Silva PH, Lima ML, Moreira RD, Souza WV, Cabral AP. Spatial study of mortality in motorcycle accidents in the State of Pernambuco, Northeastern Brazil. Rev Saude Publica 2011; 45(2):409-415.

49. Oliveira NLB, Sousa RMC. Fatores associados ao óbito de motociclistas nas ocorrências de trânsito. Rev Esc Enferm USP 2012; 46(6):1379-1386.

50. Paixão LMMM, Gontijo ED, Drumond EF, Friche AAL, Caiaffa WT. Acidentes de trânsito em Belo Horizonte: o que revelam três diferentes fontes de informações, 2008 a 2010. Rev Bras Epidemiol 2015; 18(1):108-122.

51. Marín-León L, Belon AP, Barros MBA, Almeida SDM, Restitutti MC. Tendência dos acidentes de trânsito em Campinas, São Paulo, Brasil: importância crescente dos motociclistas. Cad Saude Publica 2012; 28(1):3951.
52. Seerig LM, Bacchieri G, Nascimento GG, Barros AJD, Demarco FF. Use of motorcycle in Brazil: users profile, prevalence of use and traffic accidents occurrence - a population-based study. Cien Saude Colet 2016; 21(12):3703-3710.

53. Silva ER, Cardoso BC, Santos MPS. O aumento da taxa de motorização de motocicletas no Brasil. Revista Brasileira de Administração Científica 2011; 2(2):4963.

54. Vieira Filho JER. Expansão da fronteira agropecuária brasileira: Desafios estruturais logísticos. In: Instituto de Pesquisa Econômica Aplicada (IPEA). Boletim regional, urbano e ambiental. Brasília: IPEA; 2015. p. 37-48.

55. Caçador SB, Monte EZ. Impactos das rendas petrolíferas no crescimento econômico dos municípios no Espírito Santo. Rev Econ 2013; 39(1):129-148.

56. Noland R. Medical treatment and traffic fatality reductions in industrialized countries. Accid Anal Prev 2003; 35(6):877-883.

57. Pordeus AMJ, Vieira LJES, Almeida PC, Andrade LM, Silva ACG, Lira SVG. Fatores associados à ocorrência do acidente de motocicleta na percepção do motociclista hospitalizado. Rev Bras Promoç Saúde 2010; 23(3):206-212.

58. Mascarenhas MDM, Souto RMCV, Malta DC, Silva MMA, Lima CM, Montenegro MMS. Características de motociclistas envolvidos em acidentes de transporte atendidos em serviços públicos de urgência e emergência. Cien Saude Colet 2016; 21(12):3661-3671.

59. Tay R. The effectiveness of enforcement and publicity campaigns on serious crashes involving young male drivers: Are drink driving and speeding similar. Accid Anal Prev 2005; 37(5):922-929.

Artigo apresentado em 30/11/2017

Aprovado em 14/06/2018

Versão final apresentada em 16/06/2018 